\title{
Correlations between the textural features of wheat kernels and the quantity of DNA of Fusarium fungi
}

\author{
Ewa Ropelewska $^{1} \cdot$ Sebastian Jurczak ${ }^{2} \cdot$ Katarzyna Bilska $^{2} \cdot$ Tomasz Kulik $^{2} \cdot$ Piotr Zapotoczny $^{1}$
}

Received: 7 December 2018 / Revised: 3 January 2019 / Accepted: 12 January 2019 / Published online: 24 January 2019

(c) The Author(s) 2019

\begin{abstract}
The aim of this study was to determine the relationship between the surface textural features of wheat kernels and the quantity of DNA of fungi of the genus Fusarium. The images of kernels of four wheat cultivars were acquired on the ventral side and dorsal side with a flatbed scanner. Fungal DNA was quantified with specific TaqMan probes for $F$. culmorum and $F$. graminearum sensu stricto. In images acquired on the ventral side, the quantity of fungal DNA was most highly correlated with texture aHVariance from channel a in cultivar $1(0.86)$, with one texture from channel R in cultivar $2(0.80)$, with one texture from channel L (0.69) and one texture from channel X (-0.69) in cultivar 3, and with one texture from channel $b$ in cultivar 4 (0.70). In the images acquired on the dorsal side, the quantity of fungal DNA was most highly correlated with one texture from channel V in cultivar $3(0.89)$, two textures from channel B in cultivar $1(-0.74)$, one texture from channel R $(-0.77)$, and two textures from channel Z $(0.77)$ in cultivar 2 , and one texture from channel a and textures from channel S $(0.74)$ in cultivar 4 . The results of this study indicate the usefulness of image analysis for detecting fungal infections in grain. The findings make a valuable contribution to the development of rapid, inexpensive, non-invasive, and effective methods for preliminary screening of infected and healthy kernels and for evaluating the severity of fungal infections in kernels.
\end{abstract}

Keywords Wheat kernels $\cdot$ Textures $\cdot$ Fusarium $\cdot$ DNA quantity

\section{Introduction}

Fusarium head blight (FHB), also known as scab, is one of the most devastating diseases of small-grain cereals worldwide. FHB affects barley, rye, oats, and triticale, but it poses the greatest risk for wheat on the global scale [1]. The disease is favored by warm and humid weather at flowering $[2$, 3]. Symptoms of FHB are usually observed across the entire head or on several spikelets, and they lead to the formation of Fusarium damaged kernels (FDK). The affected grain

Electronic supplementary material The online version of this article (https://doi.org/10.1007/s00217-019-03240-0) contains supplementary material, which is available to authorized users.

Ewa Ropelewska

ewa.ropelewska@uwm.edu.pl

1 Department of Systems Engineering, Faculty of Engineering, University of Warmia and Mazury in Olsztyn, Heweliusza 14, 10-718 Olsztyn, Poland

2 Department of Botany and Nature Protection, University of Warmia and Mazury in Olsztyn, Plac Łódzki 1, 10-727 Olsztyn, Poland accumulates various mycotoxins, which significantly compromises food and feed safety [4] and poses a considerable risk for human and animal health $[5,6]$. The disease can induce changes in the shape and color of Fusarium-infected kernels which become shriveled with pink, chalky white, orange, bluish black, black, and bronze discoloration. Sporodochia and perithecia are visible on the surface of infected kernels. The disease can also reduce kernel weight $[7,8]$.

Fungal infections can decrease the quantity and quality of grain yields [9]. Grain colonized by Fusarium fungi can also accumulate mycotoxins. Type B trichothecenes, including deoxynivalenol (DON) and its acetylated derivatives (3AcDON, 15AcDON), nivalenol (NIV) and fusarenon $\mathrm{X}$ (FUS-X) and zearalenone (ZEA), are most prevalent in Europe [9-11]. Molecular and chemical methods and techniques are most commonly used to identify infected kernels. However, these methods are expensive, time-consuming, and laborious. Molecular methods based on real-time polymerase chain reaction (PCR) support the quantification of fungal species [12]. Infected kernels and mycotoxins can also be detected with the use of chemical methods, including thin-layer chromatography, gas chromatography, liquid 
chromatography, gas chromatography-mass spectrometry, liquid chromatography-mass spectrometry, fluorescence polarization immunoassay, and the enzyme-linked immunosorbent assay $[8,12,13]$. In a study by Jin et al. [8], DON concentrations estimated by gas chromatography-mass spectrometry were correlated with the results obtained by single-kernel near-infrared (SKNIR) reflectance, and SKNIRestimated FDK were additionally compared with visually estimated FDK. Spectroscopic methods are used to detect fungal infections based on the ergosterol content of kernels [12]. Ergosterol content can also be estimated on the basis of thermal properties [14]. Fungal cultures can be inspected under a microscope or with the naked eye [15], but these methods are time-consuming and require extensive mycological expertise [16]. Infected kernels can also be detected with the use of imaging techniques. Thermal imaging supports the classification of infected and healthy kernels [16, 17]. Image analysis is a non-destructive method of identifying fungal infections in kernels [12]. Delwiche et al. [18], Shahin and Symons [19], and Barbedo et al. [5] relied on hyperspectral imaging to classify Fusarium-infected and healthy kernels. Digital image analysis was used to distinguish healthy and damaged kernels with the use of color descriptors [20], shape factors [21], and textural parameters [22]. There is a general scarcity of published information about the use of textural parameters for evaluating the quantity of Fusarium DNA in infected kernels. Rapid, inexpensive, and effective methods for preliminary grain screening are, therefore, needed to distinguish infected and healthy kernels, and assess the severity of fungal infections in kernels. The aim of this study was to determine the relationship between the surface textural features of wheat kernels and the quantity of DNA of fungi of the genus Fusarium, and to identify textures that are most correlated with fungal DNA. A novel non-invasive diagnostic method was proposed for preliminary detection of fungal infections in grain and for determining the amount of fungal DNA based on kernel textures.

\section{Materials and methods}

\section{Materials}

The experiment was performed on the grain of four wheat cultivars. Grain samples were collected on two farms in northern Poland (two cultivars on one farm and two cultivars on the second farm). The samples of each cultivar were collected at random from the mass of the grain. A single sample was taken from five places of the whole available mass of grain. The individual subsamples were combined to obtain $5 \mathrm{~kg}$ of grain of each cultivar. Fusarium damaged kernels (FDK) were manually selected from bulk samples. Exemplary infected kernels are presented in Fig. 1.

\section{Image analysis}

The infected kernels were scanned individually on dorsal and ventral sides with the use of the Epson Perfection 4490 Photo flatbed scanner (UK) and SilverFast Ai Studio Epson v6.6.1r6 scanning software (LaserSoft Imaging, Inc., USA).
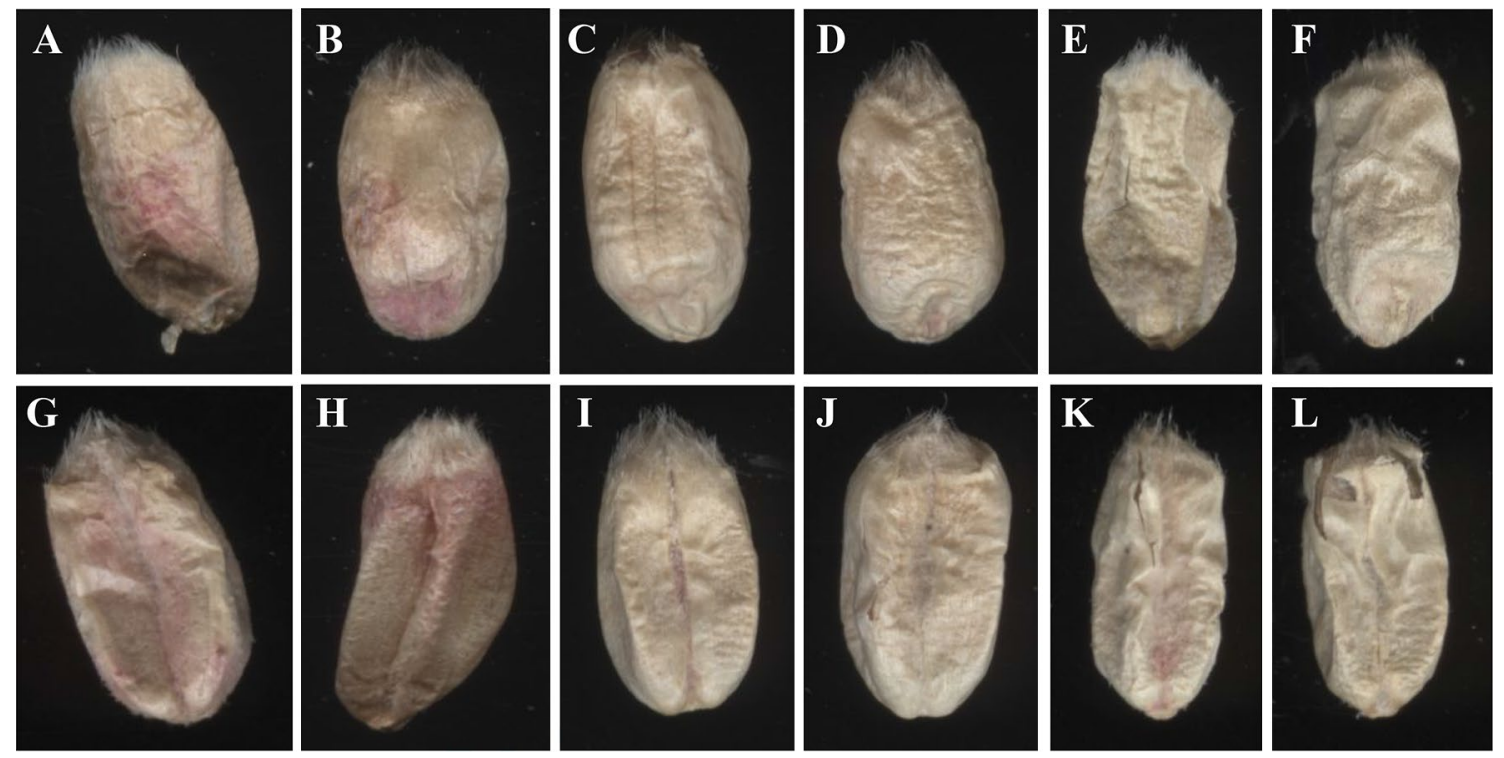

Fig. 1 Infected wheat kernels: a, b chalky white with pink discoloration, dorsal side; $\mathbf{c}, \mathbf{d}$ chalky white discoloration, dorsal side; $\mathbf{e}, \mathbf{f}$ shriveled kernels, chalky white discoloration, dorsal side; $\mathbf{g}$, h chalky white with pink discoloration, ventral side; $\mathbf{i}, \mathbf{j}$ chalky white discoloration, ventral side; $\mathbf{k}, \mathbf{I}$ shriveled kernels, chalky white discoloration, ventral side 
A total 100 kernels belonging to four cultivars were used in analysis. The scanner was color calibrated against a reflective IT8.7/2 target before measurement. A total of 200 kernel images were acquired, including 100 images of the dorsal side and 100 images of the ventral side. The acquired images with the size of $500 \times 700$ pixels and 1200 dpi resolution were saved in TIFF format.

The images were processed in the MaZda application (Łódź University of Technology, Institute of Electronics, Poland). The regions of interest (ROIs) composed of individual kernels positioned on the dorsal or the ventral side were determined and separated from the background of the scene. Quantitative analyses of textural features were carried out based on the histogram, gradient, run-length matrix, cooccurrence matrix, autoregressive model, and Haar wavelet transform. Textural features from color channels R, G, $\mathrm{B}, \mathrm{L}, \mathrm{a}, \mathrm{b}, \mathrm{X}, \mathrm{Y}, \mathrm{Z}, \mathrm{U}, \mathrm{V}$, and $\mathrm{S}$ were computed. The textural analysis in MaZda software was described in detail by Szczypiński et al. [23].

\section{Preparation of cell lysates for qPCR}

Single FDK were ground in liquid nitrogen with a mortar and pestle and transferred to tubes with $1 \mathrm{~mm}$ silica spheres (Lysing matrix C, MP Biomedicals). The samples were homogenized at $40 \mathrm{~s}$ at the speed $6.0 \mathrm{~m} / \mathrm{s}$ in $1 \mathrm{ml}$ of deionized water in the FastPrep-24 instrument (MP Biomedicals, Solon, $\mathrm{OH}$ ). After homogenization, $40 \mu \mathrm{l}$ of the homogenate was diluted with $160 \mu \mathrm{l}$ of water and incubated for $2 \mathrm{~min}$ at $90{ }^{\circ} \mathrm{C} .2 \mu \mathrm{l}$ of the aqueous phase was used for qPCR.

\section{Quantification of Fusarium species}

Two species-specific TaqMan probes for $F$. graminearum and $F$. culmorum sensu stricto were used to quantify the fungal DNA extracted from FDK according to the method described by Kulik et al. [10] and Bilska et al. [24]. F. graminearum and $F$. culmorum are the dominant species of fungi of the genus Fusarium, which caused Fusarium head blight (FHB) of cereals in Europe, including Poland [25]. The probe included a minor groove binder (MGB) moiety at the 3 '-end and was labeled with 6-carboxyfluorescein (FAM) at the 5'-end. The probes were obtained from ABI PRISM Primers and TaqMan Probe Synthesis Service, and the primers were synthesized by Sigma-Aldrich (St. Louis, MO, USA). The TaqMan reaction was performed according to Kulik et al. [10] in the 7500 Fast Real-Time PCR System (Applied Biosystems, Carlsbad, CA, USA). The efficiency of the TaqMan assay was determined with a tenfold serial dilution of various amounts of gDNA (in picograms) of either $F$. culmorum or $F$. graminearum as the template, and it was similar to that reported by Kulik et al. [10] and Bilska et al. [24]. Each reaction was carried out in three replicates.

\section{Statistical analysis}

Correlation analyses were carried out in Statistica 12.0 software [26] to determine the linear relationships between textural features and the quantity of DNA of Fusarium fungi in wheat kernels. Pearson's correlation coefficients $(R)$ were calculated at a significance level of $P<0.05$. Regression equations were also developed.

\section{Results and discussion}

\section{Correlation between textural features on the ventral side of wheat kernels and the quantity of fungal DNA}

The five highest correlation coefficients denoting significant relationships between the quantity of fungal DNA and the textural features on the ventral side of wheat kernels from each color channel were considered (Supplementary Table S1). The values of correlation coefficients differed across wheat cultivars and color channels. The highest correlation coefficient $(0.86)$ was noted for texture aHVariance from channel a in cultivar 1 . In cultivar 1 , the quantity of fungal DNA was not significantly correlated with any of the textures from channel S. In cultivar 2, the strongest correlation (0.80) was noted for texture RS5SH5AngScMom from channel R. No significant correlations were found for the textures from channels $b$ and $U$. In cultivar 3, the highest correlation coefficients were noted for texture LTGabZ4 from channel L (0.69) and texture XS5SZ5SumEntrp from channel X (-0.69). The quantity of fungal DNA was not significantly correlated with any textures from channels $\mathrm{G}$, a, and Z. In cultivar 4, the highest correlation was noted for texture bS4RNRLNonUni from channel b (0.70).

Scatter plots indicating the quantity of fungal DNA and selected textural features on the ventral side of wheat kernels and the highest correlation coefficients for each cultivar are presented in Fig. 2. Regression equations are presented in Table 1 . The amount of fungal DNA can be estimated based on the values of kernel textures.

\section{Correlation between textural features on the dorsal side of wheat kernels and the quantity of fungal DNA}

The textures on the dorsal side of kernels (Supplementary Table S2) were also highly correlated with the quantity of DNA of Fusarium fungi. The strongest correlations were noted for textures VSGKurtosis (0.89) and VSGSkewness $(0.86)$ from channel $\mathrm{V}$ in cultivar 3 . In cultivar 1 , the highest correlation coefficient $(-0.74)$ was observed for textures BS5SH3SumOfSqs and BS5SH5SumOfSqs 

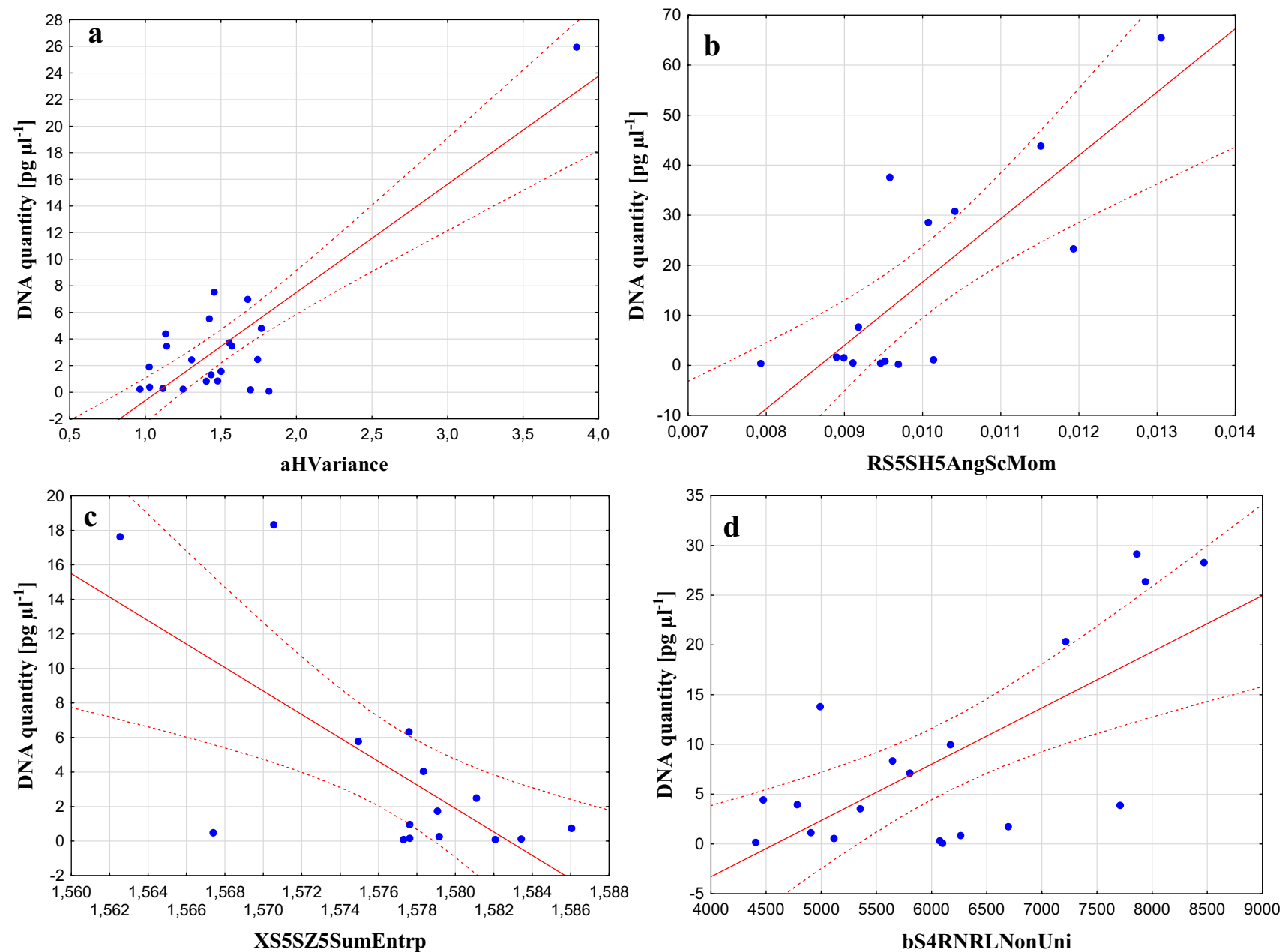

Fig. 2 Scatter plots indicating the quantity of fungal DNA and selected textural features on the ventral side of wheat kernels from cultivar 1 (a), cultivar 2 (b), cultivar 3 (c), and cultivar 4 (d)

Table 1 Regression equations of the quantity of DNA of Fusarium fungi and the textural features on the ventral and dorsal sides of wheat kernels

\begin{tabular}{llcl}
\hline Side of wheat kernels & Cultivar & $\begin{array}{l}\text { Correlation coef- } \\
\text { ficient }\end{array}$ & Regression equations \\
\hline Ventral side & 1 & 0.86 & DNA quantity $[\mathrm{pg} / \mu \mathrm{l}]=-8.745+8.1277 \times$ aHVariance \\
& 2 & 0.80 & DNA quantity $[\mathrm{pg} / \mu \mathrm{l}]=-109.9+12657.0 \times \mathrm{RS} 5 \mathrm{SH} 5 \mathrm{AngScMom}$ \\
& 3 & -0.69 & DNA quantity $[\mathrm{pg} / \mu \mathrm{l}]=1075.8-679.7 \times \mathrm{XS} 5 \mathrm{SZ5SumEntrp}$ \\
& 4 & 0.70 & DNA quantity $[\mathrm{pg} / \mu \mathrm{l}]=-25.89+0.00565 \times \mathrm{bS} 4 \mathrm{RNRLNonUni}$ \\
Dorsal side & 1 & -0.74 & DNA quantity $[\mathrm{pg} / \mu \mathrm{l}]=167.38-6.464 \times$ BS5SH3SumOfSqs \\
& 2 & -0.77 & DNA quantity $[\mathrm{pg} / \mu \mathrm{l}]=234.36-457.1 * \mathrm{RS} 4 \mathrm{RNShrtREmp}$ \\
& 3 & 0.89 & DNA quantity $[\mathrm{pg} / \mu \mathrm{l}]=-11.23+5.6414 \times$ VSGKurtosis \\
& 4 & 0.70 & DNA quantity $[\mathrm{pg} / \mu \mathrm{ll}]=-848.0+26.452 \times \mathrm{SS} 5 \mathrm{SN} 1 \mathrm{SumAverg}$ \\
\hline
\end{tabular}

from channel B. In cultivar 2, the strongest correlations were observed for texture RS4RNShrtREmp from channel $\mathrm{R}(-0.77)$ and textures ZSGSkewness and ZSGKurtosis (0.77) from channel Z. In cultivar 4 , the highest correlation coefficient (0.70) was noted for texture aHPerc10 from channel a and texture SS5SN1SumAverg from channel S. The quantity of fungal DNA was not significantly correlated with any of the textures from channels a and $\mathrm{V}$ in 
cultivar 1 and with any of the textures from channel $\mathrm{S}$ in cultivar 3 .

The scatter plots for the quantity of fungal DNA and textural features on the dorsal side of wheat kernels with the highest correlation coefficient in each cultivar are shown in Fig. 3. Regression equations are presented in Table 1.

The results of this study have expanded our knowledge about the applicability of image analysis for evaluating cereal grain. In the literature, computer image analysis has been used mainly to classify the grain of various cereal species and cultivars $[27,28]$, to identify poorly developed, shriveled, and discolored kernels damaged by fungi or insects [20-22, 29], to examine grains other than wheat [29], and to identify other materials [29]. Image analysis supported the discrimination of healthy and infected kernels with a high accuracy reaching $91 \%$ [5], 95\% [18], 90\% [19], $85 \%$ [20], 56.9-68.4\% [21], 76-100\% [22], and 85.7-100.0\%
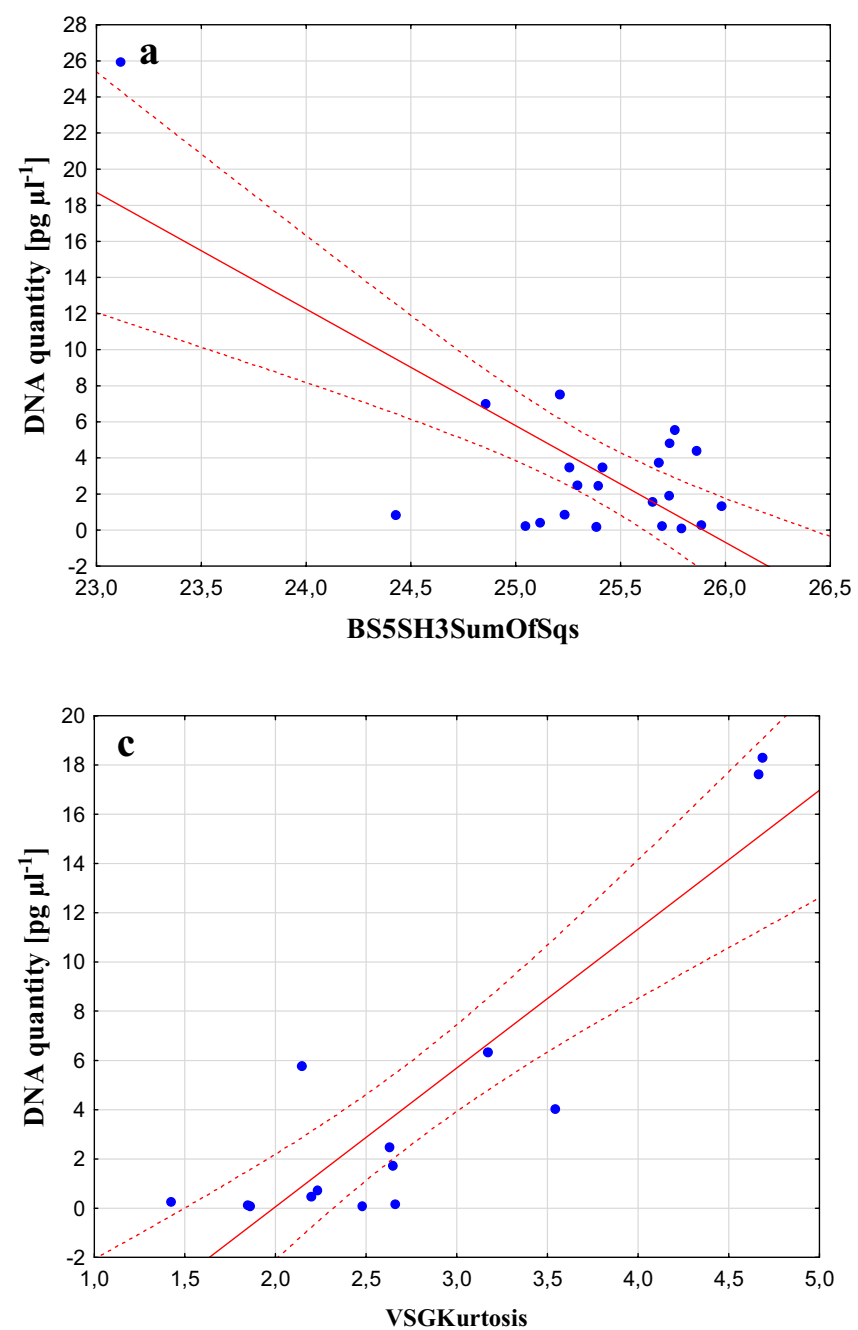

[30]. However, textural features from image analyses have been not used to estimate the quantity of DNA of Fusarium fungi. Therefore, our study has contributed new knowledge about the detection of fungal pathogens in grain.

\section{Conclusions}

High correlations were determined between the surface textural features of wheat kernels in images acquired with a flatted scanner and the quantity of DNA of fungi of the genus Fusarium. The values of correlation coefficients can differ, depending on wheat cultivar, color channel, and the side of the analyzed kernels (ventral or dorsal). The highest values of the correlation coefficient were determined at 0.86 on the ventral side and at 0.89 on the dorsal side of kernels. The amount of fungal DNA was estimated with the use of
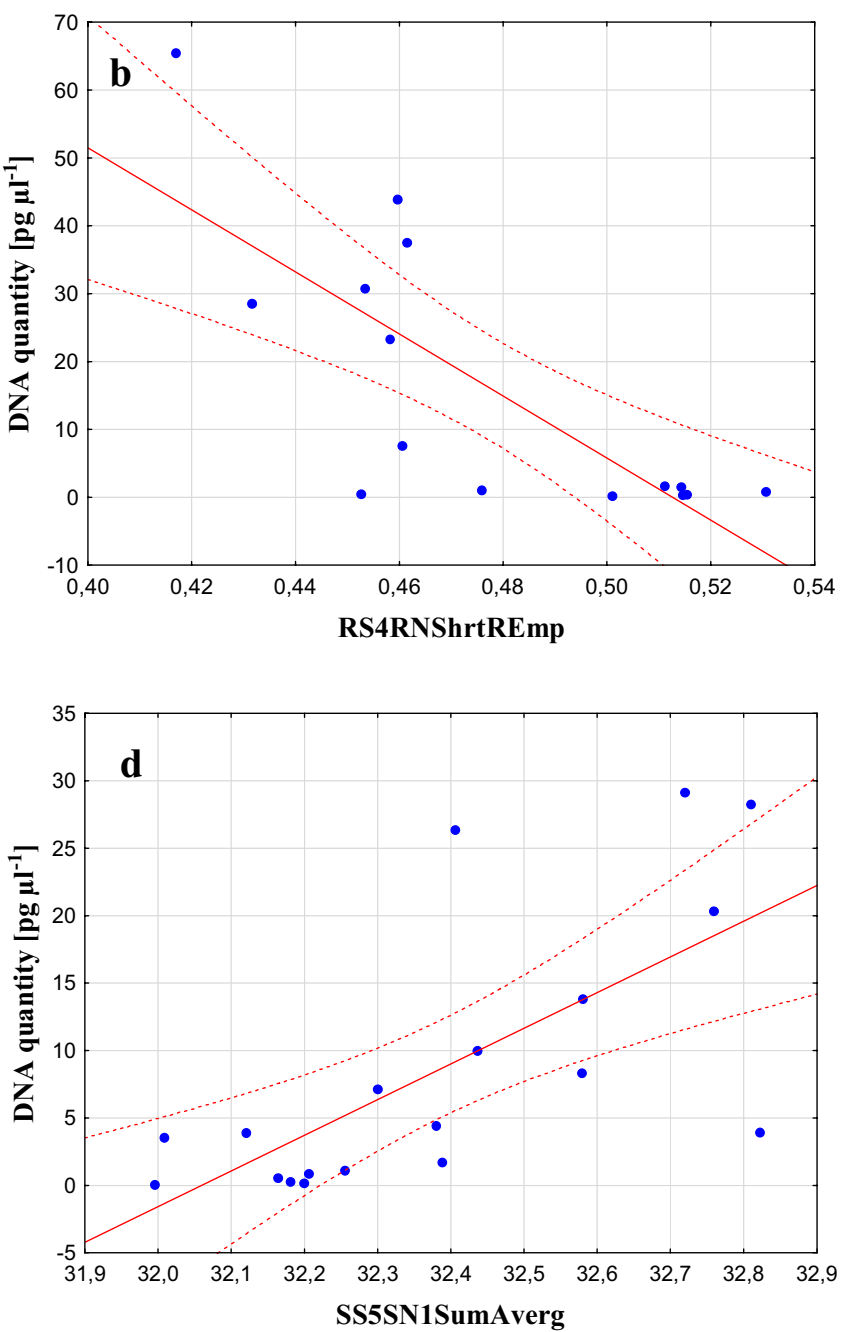

Fig. 3 Scatter plots for the quantity of fungal DNA and selected textural features on the dorsal side of wheat kernels in cultivar 1 (a), cultivar 2 (b), cultivar 3 (c), and cultivar 4 (d) 
regression equations based on textural features. The results of this study indicate that image analysis is a useful method for detecting fungal infections in grain. Our findings make a valuable contribution to the development of rapid, inexpensive, non-invasive, and effective methods for preliminary screening of infected kernels and for evaluating the severity of fungal infections in kernels.

Acknowledgements This study was conducted as part of a comprehensive research project financed by the University of Warmia and Mazury in Olsztyn (grant No. 16.620.014-300, grant No. 16.620.019-300). The study was funded by the National Science Center, Poland (Grant number 2015/19/B/NZ9/01329) and supported by the National Centre for Research and Development, Poland (Grant No. PBS3/A8/38/2015).

\section{Compliance with ethical standards}

Conflict of interest The authors declare no conflict of interest associated with this publication.

Compliance with ethics requirements This article does not contain any studies with human participants or animals performed by any of the authors.

OpenAccess This article is distributed under the terms of the Creative Commons Attribution 4.0 International License (http://creativeco mmons.org/licenses/by/4.0/), which permits unrestricted use, distribution, and reproduction in any medium, provided you give appropriate credit to the original author(s) and the source, provide a link to the Creative Commons license, and indicate if changes were made.

\section{References}

1. Figueroa M, Hammond-Kosack KE, Solomon PS (2018) A review of wheat diseases-a field perspective. Mol Plant Pathol 19(6):1523-1536

2. Doohan FM, Brennan J, Cooke BM (2003) Influence of climatic factors on Fusarium species pathogenic to cereals. Eur J Plant Pathol 109:755-768

3. Wegulo SN, Baenziger PS, Hernandez Nopsa J, Bockus WW, Hallen-Adams H (2015) Management of Fusarium head blight of wheat and barley. Crop Prot 73:100-107

4. Desjardins AE (2006) Fusarium Mycotoxins Chemistry. Genetics and Biology. American Phytopathological Society Press, St. Paul

5. Barbedo JGA, Tibola CS, Fernandes JMC (2015) Detecting Fusarium head blight in wheat kernels using hyperspectral imaging. Biosyst Eng 131:65-76

6. Barbedo JGA, Tibola CS, Lima MIP (2017) Deoxynivalenol screening in wheat kernels using hyperspectral imaging. Biosyst Eng 155:24-32

7. Symons SJ, Clear RM, Bell K, Butler C (2002) Identifying wheat and barley seed affected by Fusarium head blight. Grain biology bulletin No. 2 (3rd edn) Grain Research Laboratory, Canadian Grain Commission, Winnipeg

8. Jin F, Bai G, Zhang D, Dong Y, Ma L, Bockus W, Dowell F (2014) Fusarium-damaged kernels and deoxynivalenol in Fusariuminfected U.S. winter wheat. Phytopathology 104(5):472-478

9. Suproniene S, Sakalauskas S, Stumbriene K, Zvirdauskiene R, Svegzda P (2016) Variances in trichothecene chemotype distribution in Lithuanian wheat grain and within pure culture Fusarium graminearum isolated from the same grain samples. Eur J Plant Pathol 144:371-381

10. Kulik T, Ostrowska A, Buśko M, Pasquali M, Beyer M, Stenglein S, Załuski D, Sawicki J, Treder K, Perkowski J (2015) Development of an FgMito assay: a highly sensitive mitochondrial based qPCR assay for quantification of Fusarium graminearum sensu stricto. Int J Food Microbiol 210:16-23

11. Karlsson I, Friberg H, Kolseth AK, Steinberg Ch, Persson P (2017) Agricultural factors affecting Fusarium communities in wheat kernels. Int J Food Microbiol 252:53-60

12. Polder G, van der Heijden GWAM, Waalwijk C, Young IT (2005) Detection of Fusarium in single wheat kernels using spectral imaging. Seed Sci Technol 33:655-668

13. Meneely JP, Ricci F, van Egmond HP, Elliott ChT (2011) Current methods of analysis for the determination of trichothecene mycotoxins in food. TrAC Trend Anal Chem 30(2):192-203

14. Ropelewska E (2018) Relationship of thermal properties and ergosterol content of barley grains. J Cereal Sci 79:328-334

15. Levasseur-Garcia C (2012) Infrared spectroscopy applied to identification and detection of microorganisms and their metabolites on cereals (corn, wheat, and barley). In: Aflakpui DG (ed) Agricultural science. InTech, Rijeka, Croatia, pp 185-196

16. Chelladurai V, Jayas DS, White NDG (2010) Thermal imaging for detecting fungal infection in stored wheat. J Stored Prod Res 46:174-179

17. Kheiralipour K, Ahmadi H, Rajabipour A, Rafiei S, JavanNikkhah M (2014) Classifying healthy and fungal infected-pistachio kernel by thermal imaging technology. Int J Food Prop 18(1):93-99

18. Delwiche SR, Kim MS, Dong Y (2011) Fusarium damage assessment in wheat kernels by Vis/NIR hyperspectral imaging. Sens Instrum Food Qual Saf 5(2):63-71

19. Shahin MA, Symons SJ (2012) Detection of fusarium damage in Canadian wheat using visible/near-infrared hyperspectral imaging. J Food Meas Charact 6:3-11

20. Jirsa O, Polišenska I (2011) Identification of Fusarium damaged wheat kernels using image analysis. Acta Univ Agric Silvic Mendelianae Brun 59:125-130

21. Menesatti P, Antonucci F, Costa C, Santori A, Niciarelli I, Infantino A (2009) Application of morphometric image analysis system to evaluate the incidence of Fusarium head blight wheat infected kernels. In: Proceedings of Bornimer Agrartechnische BerichteHeft, Potsdam, Berlin, Germany, pp 157-161

22. Ropelewska E, Zapotoczny P (2018) Classification of Fusariuminfected and healthy wheat kernels based on features from hyperspectral images and flatbed scanner images: a comparative analysis. Eur Food Res Technol 244(8):1453-1462

23. Szczypiński PM, Strzelecki M, Materka A, Klepaczko A (2009) MaZda - A software package for image texture analysis. Comput Meth Prog Biol 94(1):66-76

24. Bilska K, Kulik T, Ostrowska-Kołodziejczak A, Buśko M, Pasquali M, Beyer M, Baturo-Cieśniewska A, Juda M, Załuski D, Treder K, Denekas J, Perkowski J (2018) Development of a Highly Sensitive FcMito qPCR Assay for the Quantification of the Toxigenic Fungal Plant Pathogen Fusarium culmorum. Toxins 10:211

25. Bilska K, Jurczak S, Kulik T, Ropelewska E, Olszewski J, Żelechowski M, Zapotoczny P (2018) Species composition and trichothecene genotype profiling of Fusarium field isolates recovered from wheat in Poland. Toxins 10(8):325:1-14

26. STATISTICA (Data Analysis Software System) (2013) Version 12.0. StatSoft. Inc., Tulsa

27. Majumdar S, Jayas DS (2000) Classification of cereal grains using machine vision: II. Color models. T ASAE 43(6):1677-1680 
28. Szczypiński PM, Klepaczko A, Zapotoczny P (2015) Identifying barley varieties by computer vision. Comput Electron Agric 110:1-8

29. Sun DW (2010) Hyperspectral imaging for food quality analysis and control. Academic Press/Elsevier, San Diego, pp 1-477
30. Singh CB, Jayas DS, Paliwal J, White NDG (2012) Fungal damage detection in wheat using short-wave near-infrared hyperspectral and digital colour imaging. Int J Food Prop 15:11-24

Publisher's Note Springer Nature remains neutral with regard to jurisdictional claims in published maps and institutional affiliations. 